\title{
Three-dimensional FEM analysis of stress distribution in dynamic maxillary canine movement
}

\author{
JING Yan ${ }^{1}$, HAN XiangLong ${ }^{1}$, CHENG BiHuan ${ }^{2} \&$ BAI Ding ${ }^{3 *}$ \\ ${ }^{1}$ State Key Laboratory of Oral Diseases, Department of Orthodontics, West China Hospital of Stomatology, Sichuan University, \\ Chengdu 610041, China; \\ ${ }^{2}$ MENG EN Dental Clinic, Shenzhen 518038, China; \\ ${ }^{3}$ State Key Laboratory of Oral Diseases, Department of Orthodontics, West China Hospital of Stomatology, Sichuan University, \\ Chengdu 610041, China
}

Received October 22, 2012; accepted December 5, 2012; published online May 13, 2013

\begin{abstract}
An alteration in stress/strain distribution within periodontal ligament (PDL) occurs when the tooth is mechanical stimulated in orthodontic treatment. The majority of previous finite element studies were confined to a static evaluation on initial loading status, and a dynamic long-term analysis was rarely performed in orthodontics. The present study sought to analyze the stress distribution patterns within tooth root, PDL, and alveolar bone during dynamic canine tipping and bodily movement with three-dimensional finite element method in four consecutive therapeutic weeks. We observed that the highest stress was on root surface, secondly alveolar bone, and finally, PDL for either tipping or bodily movement. Throughout the PDL and root surface, the highest stress was always around the cervical area in both patterns of movement through four phases. Moreover, a lower and more uniform stress distribution was produced during bodily movement compared with tipping movement. These results indicated that canine translation might be healthier for periodontal tissue than tipping movement; the cervical region bore a greater load and might also be a susceptible area for tissue damage (except for the apex) regardless of movement pattern.
\end{abstract}

dynamic tooth movement, finite element method (FEM), stress distribution

Citation: Jing Y, Han X L, Cheng B H, et al. Three-dimensional FEM analysis of stress distribution in dynamic maxillary canine movement. Chin Sci Bull, 2013, 58: 2454-2459, doi: 10.1007/s11434-013-5729-y

During the orthodontic tooth movement, the applied mechanical force leads to alveolar bone resorption and deposition on the pressure and tension side, respectively. Orthodontic forces trigger the biologic reactions in associated periodontal tissue. This signal transformation, which involves the processes of typical mechanotransduction for bone modeling and remodeling, includes mechanocoupling, biomechanical coupling, cell-to-cell signaling, and effector response [1]. In this way, tooth can be moved to a more appropriate position in the arch. From a mechanical point of view, on the other hand, an alteration in stress/strain distribution within periodontal ligament (PDL) and associated dentoalveolar bone occurs when the tooth is mechanical stimulated in orthodontic treatment $[2,3]$.

*Corresponding author (email: baiding@ @scu.edu.cn)
After Farah et al. [4] brought the finite element method (FEM) into dental biomechanical research in 1973, this useful technique has been extensively applied to stress/ strain analysis. Since the finite element method (FEM) can be used to analyze solids of irregular geometry that contains heterogeneous material properties, thus, it is ideally suitable to evaluate the structural behavior of tooth [5-10]. In orthodontics, finite element model has been frequently used to simulate the force application, in order to investigate the influence of alveolar bone loss, the change of center of rotation, the root resorption and stress/strain distribution during tooth movement [11-17]. At present, the majority of previous finite element studies about orthodontic tooth movement were confined to a static evaluation on initial loading status due in part to the complexity of biomechanical reaction, and a dynamic long-term finite element analysis was 
rarely preformed. Nevertheless, orthodontic tooth movement is not a one-step process. It includes four phasesinitial phase, arrest phase, acceleration phase and constant linear movement phase. Each step is associated with particular biologic reaction. Therefore, the static analysis can not reveal the genuine orthodontic tooth movement. Moreover, orthodontic force is continuous and changing along with variational tooth position. Therefore, it is of significance to understand the biomechanical response of teeth to therapeutic loads together with actual morphological and histological changes during tooth movement. In this way, orthodontic treatment could be more reliable and more effective.

One of the most common tooth movements in orthodontic treatment is canine retraction, which is a time-consuming stage in cases of first premolar extraction. Previous studies have focused on the comparison of force magnitude, the relationship between the $\mathrm{M} / \mathrm{F}$ ratio and the center of rotation, anchorage loss, tooth movement rate, and appliance efficiency [18-22]. However, whether one type of tooth movement is physiologically healthy and appropriate depends on whether it can lead to a well biological response within periodontal tissue. An optimal orthodontic force, which is decided by the pattern of stress distribution in periodontal tissue, can make the tooth movement process smoothly with minor irreversible tissue damage. On the contrary, not only will the tooth stop moving normally, but also negative consequences will occur if the orthodontist knows little about the mechanical responses for different types of tooth movement [23]. These always lead to decreased tooth function and disappointing esthetic outcome.

In orthodontic therapy, canine distalization is usually accomplished through two types of movement: tipping or translation. With the employment of three-dimensional FEM, the aim of this study was to investigate the dynamic stress distribution within tooth root, PDL, and alveolar bone by simulating tipping and bodily canine movement, respectively. Our previous study has shown that stress distribution patterns at compressive and tensile sides were similar in each phase, regardless of tipping or bodily movement during canine distalization [24]. In this paper, particular emphases will be placed on the discrepancy of stress distribution in distal side during the two patterns of movement.

\section{Material and methods}

\subsection{Generation of finite element model}

A finite element model of a maxillary canine from our previous experiment was used [24]. The three-dimensional model of maxillary canine, which has a root length of 16 $\mathrm{mm}$ and crown height of $11 \mathrm{~mm}$, was developed according to the computerized tomographic (CT) images of an extracted maxillary canine with normal geometry. The CT images consisted of 54 transversal sections at intervals of $0.5 \mathrm{~mm}$. The three-dimensional model, which was com- prised of tooth, PDL, and alveolar bone, was reconstructed and analyzed with ANSYS5.7 (Swanson Analysis System Co., Houston, TX, USA). The PDL was simulated as a 0.25 $\mathrm{mm}$ layer around the tooth root. The mechanical properties of tooth, PDL, and alveolar bone were assumed to be linear elastic, homogeneous, and isotropic. They were defined according to the experimental data in Tanne's study [5] as shown in Table 1 . The finite element model of the maxillary canine segment consisting of 45075 nodes and 32745 solid elements was thus generated (Figure 1(a)).

Solid 92 (free mesh) was adopted in the model (Figure 1(b)). It is a three-dimensional tetrahedral unit with 10 nodes, characterized by quadratic displacement and is especially fit for mesh generation of an abnormal geometric body. Each node has three degrees of freedom, symbolizing displacement in the direction of $X, Y$ and $Z$, respectively.

Table 1 Mechanical property of tooth, periodontal ligament (PDL), and alveolar bone

\begin{tabular}{lcc}
\hline Material & Young's modulus $\left(\mathrm{kg} / \mathrm{mm}^{2}\right)$ & Poisson's ratio \\
\hline Tooth & $2.0 \times 10^{3}$ & 0.15 \\
PDL & $6.8 \times 10^{-2}$ & 0.49 \\
Alveolar bone & $1.4 \times 10^{3}$ & 0.15 \\
\hline
\end{tabular}

(a)

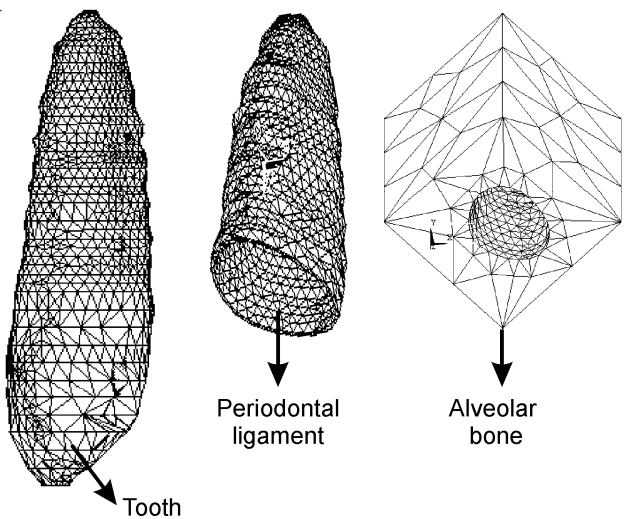

(b)

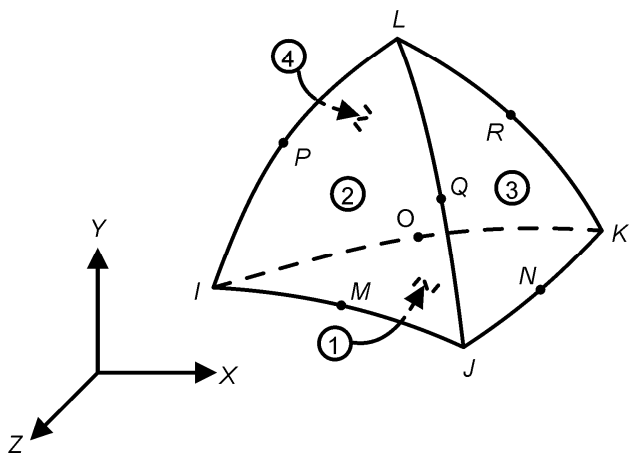

Figure 1 A three-dimensional finite element model of maxillary canine, consisting of tooth, periodontal ligament (PDL), and alveolar bone (a). A Solid 92 element is a 3D tetrahedron unit with 10 nodes. (1)-(4) represent the four planes of the tetrahedron unit, and $I-R$ represent the 10 nodes of the tetrahedron unit. Each node has three degrees of freedom, symbolizing displacement in the direction of $X, Y$ and $Z$, respectively (b). 


\subsection{Loading and boundary conditions}

Tipping movement was simulated by applying a mesiodistal-directed force $(\mathrm{F})$ of $1.47 \mathrm{~N}(150 \mathrm{~g})$ to the midpoint of the labial surface of the tooth crown, $6 \mathrm{~mm}$ apical to the incisal edge. For bodily movement, in addition to a force of $1.47 \mathrm{~N}$ in mesio-distal direction, the anti-tipping moment (M1) of $1.52 \times 10^{-2} \mathrm{~N} \mathrm{~m}$ and anti-rotation moment (M2) of $1.23 \times 10^{-2} \mathrm{~N} \mathrm{~m}$ were complemented (Figure 2(a)). As for boundary conditions, the exterior cross-sections of the alveolar bone were fixed.

\subsection{Simulation of tooth movement processes}

The orthodontic loads gradually decrease along with tooth movement. Therefore, loads were decreased $10 \%$ per week and applied at the centre of the tooth crown in simulation of both tipping and bodily movements, following the methods of Burstone [25] and Angolkar et al. [26].

A period of orthodontic treatment is usually divided into four phases, each lasting one week. Therefore, the four phases were begun at: the 0th day (P1), the 7th day (P2), the 14th day (P3) and the 21st day (P4). The process used to simulate dynamic orthodontic tooth movement went as follows [24]: (1) We established the initial pattern of stress distribution; and (2) based on this initial scenario, we calculated tooth displacement of each boundary node for each

(a)

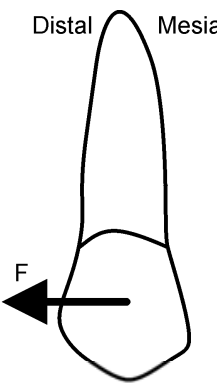

Tipping movement

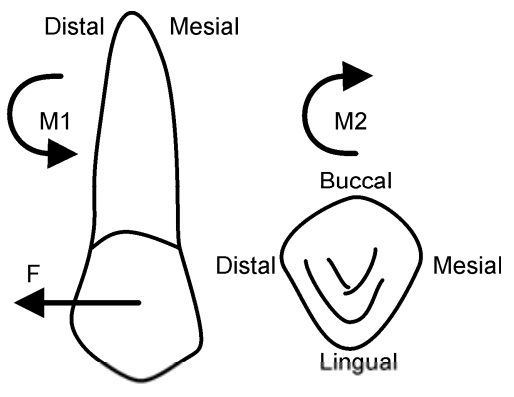

Bodily movement (b)

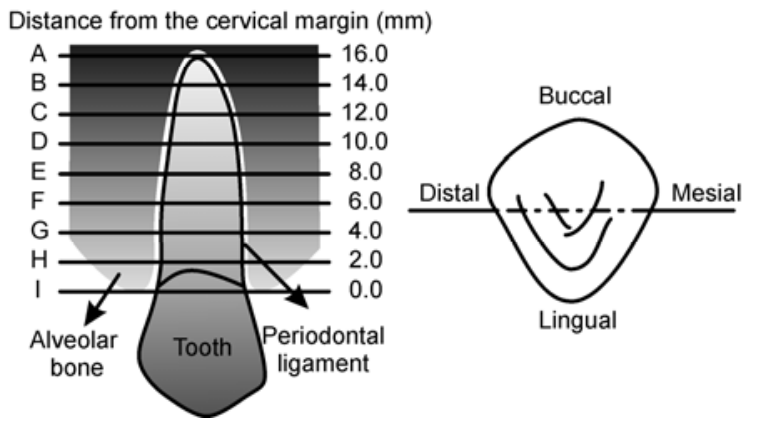

Figure 2 Two force modalities. Tipping movement: a distal horizontal force $(\mathrm{F})$ of $1.47 \mathrm{~N}(150 \mathrm{~g})$ was applied at the midpoint of the canine's labial crown (6 $\mathrm{mm}$ to the edge); bodily movement: besides a force $(\mathrm{F})$ of $1.47 \mathrm{~N}$ in mesio-distal direction, a counterclockwise moment (M1) of 1.52 $\times 10^{-2} \mathrm{~N}$ m and a clockwise moment (M2) of $1.23 \times 10^{-2} \mathrm{~N}$ m were added to counteract tipping and rotation, respectively (a). Occluso-gingival levels (A through I, interval distance of $2.0 \mathrm{~mm}$ ) and horizontal midpoints where the Von Mises stresses were determined (b). unit under normal stress (without considering shear stress). For example, we designated a layer, found all nodes, and calculated the displacement. When a node was included in more than one unit, weighted average displacement was used to calculate the final three-dimensional coordinates. In actuality, all the nodes of the same layer may not exhibit the exact same displacement value. However, as a rigid body, the tooth did not allow for separate movement of its various parts. It is necessary to distinguish the node with the minimal displacement in each layer, compare displacement of the layers, and determine the displacement for the whole tooth. Thus, a dynamic three-dimensional finite element model, which could be used to simulate continuous and dynamic tooth movement, was established successfully. The method used for the reconstruction of model is mentioned in Appendix.

Results were obtained with ANSYS, and Von Mises equivalent stress was used as the major index. The root was bisected by an imaginary buccolingual centric plane. On this plane, stress values at 9 (A-I) occluso-gingival levels $(0,2,4,6,8,10,12,14$ and $16 \mathrm{~mm})$ from the cervical crest were computed and output (Figure 2(b)). The results were graphically represented.

\section{Results and discussion}

\subsection{Finite element analysis}

Since stress or strain in living tissues is thought to be a key factor in biological change, it is important to elucidate the stress or strain to understand its relationship to bone remodeling [27]. The three-dimensional finite element method, a powerful noninvasive tool of numerical stress analytical technique, can simulate orthodontic force system applied clinically and allow analysis of the response of tooth, PDL and alveolar bone to the orthodontic loads at any given point theoretically. Plenty of literatures are available on the study of stress distribution and deformation of nonliving as well as natural or restored craniofacial structures which are susceptible to three-dimensional stress [9,27-30]. However, they were all confined to static analysis or initial stage that was the first phase in this study. The displacement of the tooth can be visualized graphically through four therapeutic phases. Furthermore, mechanical stimulation can easily be simulated through the changes of application point, magnitude, and direction of a force [31]. In the present finite element model, advanced dual-slice spiral CT was used to collect anatomical data from a human tooth. All the materials' mechanical parameters were previously set up and were in accordance with those in most of the relevant studies $[5,32,33]$. The $150 \mathrm{~g}$ mesiodistal-directed force was chosen based on accordance with clinical practice $[21,34,35]$. The results of the present study provided some additional explanation about the tooth, periodontal and bony tissue mechanical reactions, which were tentative to explore tissue 
response to the compound process of tooth movement.

\subsection{Calculation assumption}

As described before [24], the most difficult task of this study was the reconstruction of the dynamic finite element model during four different phases, which meant the development of an algorithm to simulate the constant changes in stress pattern and distribution when the tooth was moving. We adopted the above-mentioned approach to simulate the process of dynamic canine distalization. Despite the application of decreased loads in the process of dynamic tooth movement, the finite element model, of course, was still far from the real nature without considering such factors as haemodynamics of the PDL, the presence of hyalinized bone and undermined bone resorption. However, this model was completely competent for the present work, because our primary aim focused on investigating the stress distribution and changing rather than predicting the biological reaction.

\subsection{Stress distribution}

According to our results, the highest stresses were observed on root surface, alveolar bone, and PDL, subsequently, in both tipping and bodily movement. On the root surface, the highest stresses concentrated on the cervical area and declined towards the apex. Stress distribution in PDL was similar to that of the root surface, although the magnitude was lower and less regular. In the alveolar bone, the highest stresses were found at the cervical third and the apical third with a gradual reduction (to nearly 0 ) at the middle third (Figure 3). These outcomes were consistent with Tanne's study [5].

The highest stress distribution was always around the cervical area on root surface and in PDL through the four phases during tipping movement (Figure 3). In translation movement, on the other hand, the stress distributed more even throughout the PDL and the root (Figure 3). These results are in agreement with the studies by Rudolph et al. [9], and Field et al. [23]. Chan et al. [17] and Darendeliler et al. [36] demonstrated that root resorption might occurs more frequently in high-pressure zones after 28 days of force application. According to their data, root resorption at the alveolar region increased 2.66 times while apex showed no significant change between the light-force group (25 g) and heavy-force group (225 g) [17]. With the combination of our results, it may indicate that the cervical region bears greater load and might be one susceptible area for potential (a)

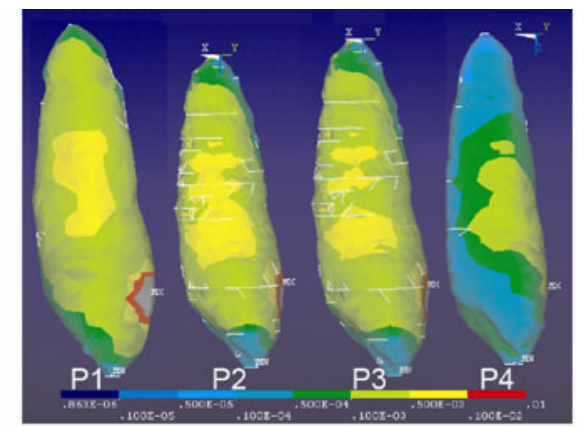

(b)

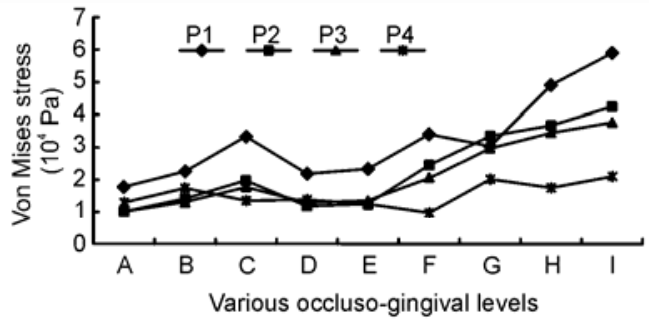

(c)

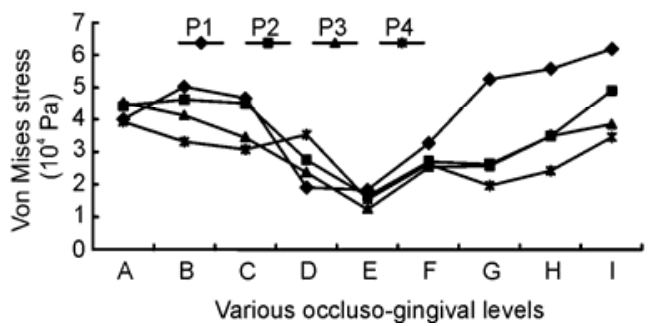

(d)
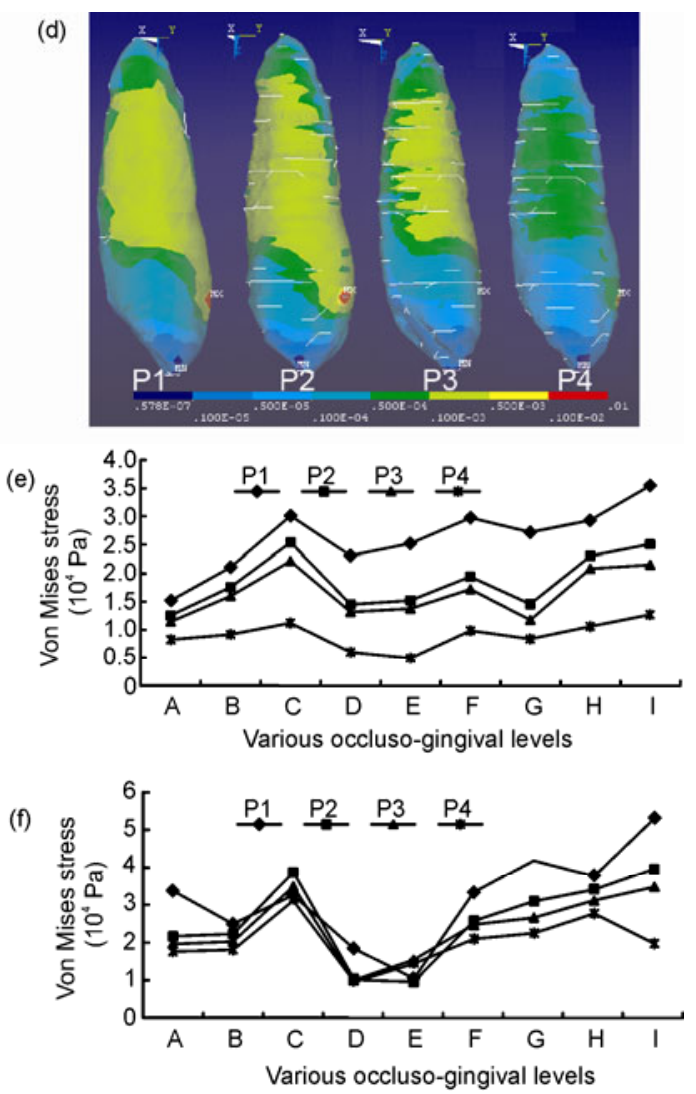

Figure 3 Stress distribution and dynamic alteration (apical up and cervical down) of root distal side during tipping (a) and bodily (d) movement at P1, P2, $\mathrm{P} 3$ and P4. The palette of colors indicates the magnitude of stress, e.g. the lowest value at the left end is $0.863 \times 10^{-6} \mathrm{~g}$ Pa and the highest value at the right end is $0.100 \times 10^{-2} \mathrm{gPa}\left(1 \mathrm{gPa}=10^{9} \mathrm{~Pa}\right)$. Stress distribution in PDL for tipping (b) and bodily (e) movement, and that in alveolar bone for tipping (c) and bodily (f) movement is shown at 9 occluso-gingival levels (A through I). 
tissue damage (except for the apex), especially in tipping movement.

Stress distribution and degradation varied with different tooth movement models (tipping and bodily movement) throughout the whole course of canine distalization. The stress degradation of bodily movement was more even, with a lower magnitude than that of tipping movement (Figure 3). The peak value of the stress in PDL for each stage during tipping movement were $0.0614,0.0524,0.0402,0.0327$ $\mathrm{MPa}$, respectively. The peak value of the stress in PDL for each stage during bodily movement was 0.0476, 0.0393, $0.0271,0.0195 \mathrm{MPa}$, respectively. It has been documented that a thick sterile necrotic area, called the hyalinized zone appears on the interface between PDL and root surface if heavy stresses concentrate in periodontal $[16,37,38]$. The higher the stress is, the bigger the hyalinized zone will be. The hyalinization can delay the rate of tooth movement and precede root resorption [16]. Therefore, bodily movement might be suggested as a healthier alternative for periodontal tissue than tipping movement. In consideration that many adult patients have periodontal problem, some researchers also suggested that orthodontic therapy in adults might be best to mostly involve pure translation movement with light forces, which could avoid overloading within the periodontal tissues $[39,40]$.

Magnitude of stress for the same locations degraded gradually during four consecutive phases (Figure 3). Overall, the areas closest to the alveolar crest decreased the fastest in terms of stress. In addition, stress in the cervical area of the alveolar bone during tipping movement degraded more quickly than during bodily movement in P1 and P2, which may have been induced by more displacement in the cervi$\mathrm{cal}$ area than in the apical area. Whether this was caused by more rapid bone resorption and remodeling during tipping movement still remains unclear and needs further research. In light of the result that the highest stress distribution was always around the cervical area within PDL and root surface in both types of movement through four phases, we suppose that it may be important to use light force to minimize tissue damage in the initial stage of orthodontic treatment if tipping movement is necessary, especially for adults who have compromised periodontal condition.

In summary, acquainted with these quantitative data, orthodontists can extend an improved understanding of the physiologic or mechanical reactions and interactions that occur within the dentoalveolar complex, thus design an optimal force system to control and obtain an appropriate type of tooth movement. Of course, in consideration of finite element model limitation, further in vivo and in vitro studies are needed to investigate the task comprehensively.

\section{Conclusion}

(1) Stress distribution and degradation varied with different tooth movement models (tipping and bodily movement) throughout the course of canine distalization. Translation produced a lower and more uniform stress distribution than tipping movement, especially in PDL.

(2) The stress in PDL during tipping movement was higher than that during bodily movement, especially in the first step. Thus, translation might be a healthier alternative for periodontal tissue than tipping movement.

(3) Throughout the PDL and root surface, the highest stress distribution was always around the cervical area in both types of movement during the four phases, indicating that cervical region, excluding the apex, bears the most loads and might be a susceptible area for potential tissue damage, regardless of movement pattern.

This work was supported by the Technology Supporting Program of Sichuan Province (2009-2011, 2009SZ0164).

1 Turner C H, Pavalko F M. Mechanotransduction and functional response of the skeleton to physical stress: The mechanisms and mechanics of bone adaptation. J Orthop Sci, 1998, 3: 346-355

2 Tanne K, Yoshida S, Kawata T, et al. An evaluation of the biomechanical response of the tooth and periodontium to orthodontic forces in adolescent and adult subjects. Br J Orthod, 1998, 25: 109-115

3 Cattaneo P M, Dalstra M, Melsen B. The finite element method: A tool to study orthodontic tooth movement. J Dent Res, 2005, 84: 428-433

4 Farah J W, Craig R G, Sikarskie D L. Photoelastic and finite element stress analysis of a restored axisymmetric first molar. J Biomech, 1973, 6: 511-520

5 Tanne K, Sakuda M, Burstone C J. Three-dimensional finite element analysis for stress in the periodontal tissue by orthodontic forces. Am J Orthod Dentofacial Orthop, 1987, 92: 499-505

6 Cobo J, Sicilia A, Arguelles J, et al. Initial stress induced in periodontal tissue with diverse degrees of bone loss by an orthodontic force: Tridimensional analysis by means of the finite element method. Am J Orthod Dentofacial Orthop, 1993, 104: 448-454

7 Vollmer D, Bourauel C, Maier K, et al. Determination of the centre of resistance in an upper human canine and idealized tooth model. Eur J Orthod, 1999, 21: 633-648

8 Qian H, Chen J, Katona T R. The influence of PDL principal fibers in a 3-dimensional analysis of orthodontic tooth movement. Am J Orthod Dentofacial Orthop, 2001, 120: 272-279

9 Rudolph D J, Willes P M G, Sameshima G T. A finite element model of apical force distribution from orthodontic tooth movement. Angle Orthod, 2001, 71: 127-131

10 Toms S R, Eberhardt A W. A nonlinear finite element analysis of the periodontal ligament under orthodontic tooth loading. Am J Orthod Dentofacial Orthop, 2003, 123: 657-665

11 Geramy A. Alveolar bone resorption and the center of resistance modification (3-D analysis by means of the finite element method). Am J Orthod Dentofacial Orthop, 2000, 117: 399-405

12 Kojima Y, Fukui H. Numerical simulation of canine retraction by sliding mechanics. Am J Orthod Dentofacial Orthop, 2005, 127: 542551

13 Kojima Y, Mizuno T, Fukui H. A numerical simulation of tooth movement produced by molar uprighting spring. Am J Orthod Dentofacial Orthop, 2007, 132: 630-638

14 Yettram A L, Wright K W, Pickard H M. Finite element stress analysis of the crowns of normal and restored teeth. J Dent Res, 1976, 55: 1004-1011

15 Vásquez M, Calao E, Becerra F, et al. Initial stress differences between sliding and sectional mechanics with an endosseous implant 
as anchorage: A 3-dimensional finite element analysis. Angle Orthod, 2001, 71: 247-256

16 Chan E, Darendeliler M A. Physical properties of root cementum: Part 7. Extent of root resorption under areas of compression and tension. Am J Orthod Dentofacial Orthop, 2006, 129: 504-510

17 Chan E, Darendeliler M A. Physical properties of root cementum: Part 5. Volumetric analysis of root resorption craters after application of light and heavy orthodontic forces. Am J Orthod Dentofacial Orthop, 2005, 127: 186-195

18 Rajcich M M, Sadowsky C. Efficacy of intraarch mechanics using differential moments for achieving anchorage control in extraction cases. Am J Orthod Dentofacial Orthop, 1997, 112: 441-448

19 Shpack N, Davidovitch M, Sarne O, et al. Duration and anchorage management of canine retraction with bodily versus tipping mechanics. Angle Orthod, 2008, 78: 95-100

20 Cattaneo P M, Dalstra M, Melsen B. Moment-to-force ratio, center of rotation, and force level: A finite element study predicting their interdependency for simulated orthodontic loading regimens. Am J Orthod Dentofacial Orthop, 2008, 133: 681-689

21 Nikolai R J. On optimum orthodontic force theory as applied to canine retraction. Am J Orthod, 1975, 68: 290-302

22 Hasler R, Schmid G, Ingervall B, et al. A clinical comparison of the rate of maxillary canine retraction into healed and recent extraction sites-A pilot study. Eur J Orthod, 1997, 19: 711-719

23 Field C, Ichim I, Swain M V, et al. Mechanical responses to orthodontic loading: A 3-dimensional finite element multi-tooth model. Am J Orthod Dentofacial Orthop, 2009, 135: 174-181

24 Zhao Z, Fan Y, Bai D, et al. The adaptive response of periodontal ligament to orthodontic force loading-A combined biomechanical and biological study. Clin Biomech (Bristol, Avon), 2008, 23(Suppl 1): S59-66

25 Burstone C J. The segmented arch approach to space closure. Am J Orthod, 1982, 82: 361-378

26 Angolkar P V, Arnold J V, Nanda R S, et al. Force degradation of closed coil springs: An in vitro evaluation. Am J Orthod Dentofacial Orthop, 1992, 102: 127-133

27 Jafari A, Shetty K S, Kumar M. Study of stress distribution and displacement of various craniofacial structures following application of transverse orthopedic forces-A three-dimensional FEM study. Angle Orthod, 2003, 73: 12-20

28 Chang Y I, Shin S J, Baek S H. Three-dimensional finite element analysis in distal en masse movement of the maxillary dentition with the multiloop edgewise archwire. Eur J Orthod, 2004, 26: 339-345

29 Gallas M M, Abeleira M T, Fernández J R, et al. Three-dimensional numerical simulation of dental implants as orthodontic anchorage. Eur J Orthod, 2005, 27: 12-16

30 Hohmann A, Wolfram U, Geiger M, et al. Periodontal ligament hydrostatic pressure with areas of root resorption after application of a continuous torque moment. Angle Orthod, 2007, 77: 653-659

31 Jafari A, Shetty K S, Kumar M. Study of stress distribution and displacement of various craniofacial structures following application of transverse orthopedic forces-A three-dimensional FEM study. Angle Orthod, 2003, 73: 12-20

32 McGuinness N J, Wilson A N, Jones M L, et al. A stress analysis of the periodontal ligament under various orthodontic loadings. Eur $\mathrm{J}$ Orthod, 1991, 13: 231-242

33 Cobo J, Sicilia A, Argüelles J, et al. Initial stress induced in periodontal tissue with diverse degrees of bone loss by an orthodontic force: tridimensional analysis by means of the finite element method. Am J Orthod Dentofacial Orthop, 1993, 104: 448-454

34 Tanne K, Inoue Y, Sakuda M. Biomechanical behavior of the periodontium before and after orthodontic tooth movement. Angle Orthod, 1995, 65: 123-128

35 Nägerl H, Burstone C J, Becker B, et al. Centers of rotation with transverse forces: An experimental study. Am J Orthod Dentofacial Orthop, 1991, 99: 337-345

36 Darendeliler M A, Kharbanda O P, Chan E K, et al. Root resorption and its association with alterations in physical properties, mineral contents and resorption craters in human premolars following application of light and heavy controlled orthodontic forces. Orthod Craniofac Res, 2004, 7: 79-97

37 Tomizuka R, Shimizu Y, Kanetaka H, et al. Histological evaluation of the effects of initially light and gradually increasing force on orthodontic tooth movement. Angle Orthod, 2007, 77: 410-416

38 von Bohl M, Kuijpers-Jagtman A M. Hyalinization during orthodontic tooth movement: A systematic review on tissue reactions. Eur J Orthod, 2009, 31: 30-36

39 Melsen B. Tissue reaction following application of extrusive and intrusive forces to teeth in adult monkeys. Am J Orthod, 1986, 89: $469-475$

40 Gunduz E, Rodriguez-Torres C, Gahleitner A, et al. Bone regeneration by bodily tooth movement: Dental computed tomography examination of a patient. Am J Orthod Dentofacial Orthop, 2004, 125: $100-106$

Open Access This article is distributed under the terms of the Creative Commons Attribution License which permits any use, distribution, and reproduction in any medium, provided the original author(s) and source are credited.

\section{Appendix}

Displacement formulas are listed as follows:

$$
\begin{aligned}
& U=U_{I}\left(2 L_{1}-1\right) L_{1}+U_{J}\left(2 L_{2}-1\right) L_{2}+U_{K}\left(2 L_{3}-1\right) L_{3}+U_{L}\left(2 L_{4}-1\right) L_{4}+4\left(U_{M} L_{1} L_{2}+U_{N} L_{2} L_{3}+U_{O} L_{1} L_{3}+U_{P} L_{1} L_{4}+U_{Q} L_{2} L_{4}+U_{R} L_{3} L_{4}\right) \\
& V=V_{I}\left(2 L_{1}-1\right) L_{1}+V_{J}\left(2 L_{2}-1\right) L_{2}+V_{K}\left(2 L_{3}-1\right) L_{3}+V_{L}\left(2 L_{4}-1\right) L_{4}+4\left(V_{M} L_{1} L_{2}+V_{N} L_{2} L_{3}+V_{O} L_{1} L_{3}+V_{P} L_{1} L_{4}+V_{Q} L_{2} L_{4}+V_{R} L_{3} L_{4}\right) \\
& W=W_{I}\left(2 L_{1}-1\right) L_{1}+W_{J}\left(2 L_{2}-1\right) L_{2}+W_{K}\left(2 L_{3}-1\right) L_{3}+W_{L}\left(2 L_{4}-1\right) L_{4}+4\left(W_{M} L_{1} L_{2}+W_{N} L_{2} L_{3}+W_{O} L_{1} L_{3}+W_{P} L_{1} L_{4}+W_{Q} L_{2} L_{4}+W_{R} L_{3} L_{4}\right) \\
& U, V \text { and } W \text { equal displacement in the direction of } X, Y \text { and } Z \text {, respectively. } L_{1}, L_{2}, L_{3} \text { and } L_{4} \text { are volume coordinates. A }
\end{aligned}
$$
random node $P$ in a unit has volume coordinates as

$$
L_{1}=\frac{\operatorname{vol}(P 234)}{\operatorname{vol}(1234)}, \quad L_{2}=\frac{\operatorname{vol}(P 341)}{\operatorname{vol}(1234)}, \quad L_{3}=\frac{\operatorname{vol}(P 412)}{\operatorname{vol}(1234)}, \quad L_{4}=\frac{\operatorname{vol}(P 123)}{\operatorname{vol}(1234)} .
$$

